\title{
Can Treatment Adherence Be Improved by Using Rubin's Four Tendencies Framework to Understand a Patient's Response to Expectations?
}

\author{
Jeremy Kirk ${ }^{\mathrm{a}} \quad$ Anita MacDonald $^{\mathrm{b}} \quad$ Paul Lavender $^{\mathrm{c}} \quad$ Jessica Dean $^{\mathrm{d}}$ \\ Gretchen Rubin ${ }^{\mathrm{e}}$ \\ ${ }^{a}$ Department of Endocrinology, Birmingham Children's Hospital, and bepartment of

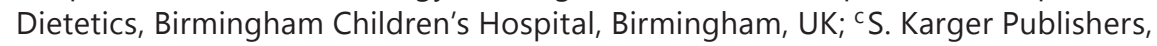 \\ Basel, Switzerland; dDepartment of Clinical Health Psychology, Salford Royal Hospital,

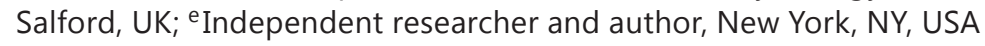

\begin{abstract}
What Is It about?
Individuals respond to expectations in different but recognisable ways, and we need to consciously take these patterns into account when engaging patients in treatment programmes. Using Rubin's Four Tendencies model to understand the way in which a patient responds to external and internal expectations of treatment adherence may provide the opportunity to develop and validate simple clinical tools to match a patient's dominant "Tendency" ("Upholder," "Questioner," "Obliger," "Rebel") to complementary interventions to increase adherence.
\end{abstract}

\section{Keywords}

Treatment adherence $\cdot$ Rubin's Four Tendencies $\cdot$ Compliance $\cdot$ Medication $\cdot$ Intervention

\begin{abstract}
Within the context of poorer patient outcomes and rising healthcare costs, we need to better understand why many patients do not engage fully with their treatment plan. Movement away from talking about "compliance" towards "adherence" and "concordance" is evidence of a recognition that this is a two-way process. Whilst healthcare professionals expect patients to engage in treatment, equally, patients have expectations (whether positive or negative) of their treatment and their need for engagement. There is a need for an effective method that can specifically target those interventions that will provide the most benefit to individual pa-
\end{abstract}

For queries relating to Rubin's Four Tendencies model, please contact Gretchen Rubin. For queries relating to the potential clinical testing of the model, please contact Jessica Dean.

Gretchen Rubin

1324 Lexington Ave. \#215

New York, NY 10128 (USA)

E-Mail gretchenrubin1@gretchenrubin.com
Jessica Dean

Salford Royal NHS Foundation Trust

Department of Clinical Health Psychology, Salford Royal Hospital

Stott Ln, Salford M6 8HD (UK)

E-Mail Jessica.Dean@srft.nhs.uk 
tients and which, crucially, is easy and inexpensive to administer in everyday practice and widely applicable. Rubin's Four Tendencies model identifies a patient's "response to outer and inner expectations" as a key factor in adherence. The model therefore provides an opportunity to test such a targeted, patient-specific strategy and we present a call to action for research in this area.

\section{What Is Adherence and Why Does It Matter?}

It would appear logical to many that if a person has an illness that causes them discomfort, pain, or even the long-term risk of serious harm or death that they would engage in treatment. But we know that adherence to treatment is not guaranteed, and that approximately half of patients do not take their medication as prescribed [1]. Patient adherence in the real world relates to a person's ability to undertake their treatment programme in a way in which they have agreed with their healthcare professional (HCP), and hopefully, in a way which we know from clinical trials can produce a benefit. Importantly, we know that these benefits are greatly reduced or nullified in usual clinical practice when adherence rates are low [2]. Clearly, if the benefits of medications that we see in the carefully controlled and monitored setting of clinical trials are to be translated into real-world effects, low patient adherence is a significant hurdle for HCPs to overcome [2].

Beyond maximising treatment benefits to individual patients, there is also a wider issue to be considered that is now fundamental to healthcare systems worldwide, that of escalating costs in the face of increasing demand [3]. Patient non-adherence can propagate a downward spiral, with the poor outcomes resulting from not taking medication increasing the need for further intervention by the healthcare service and thus escalating the ongoing costs. One thing is clear, within the context of poorer patient outcomes and rising costs, we need to better understand why many patients are not engaging fully with their treatment plan.

\section{What Factors Influence Patient Adherence?}

In a recent Cochrane Database review of interventions for the enhancement of adherence, only 17 of 182 studies included were considered to have a low risk of bias in their design, and thus reviewed in detail. Most importantly, the specific characteristics and effects of interventions identified as improving adherence varied among these studies and the authors were not certain how consistent improvements could be achieved so that the full health benefits of medicines can be realised [2]. Importantly, almost all of the interventions judged to be effective for long-term care were complex. However, even the most effective interventions did not produce large improvements in adherence and treatment outcomes [2].

The reasons that patients do not adhere to their treatment are multifaceted, results from different studies are often conflicting [4], and there is a complicated interplay between contributing factors. These factors can be grouped as patient-related, provider-related, and external (Fig. 1) [3, 5].

Research into adherence has usually focused on common barriers patients face in taking their medication, as many of these are viewed as under a patient's control and therefore modifiable, but success has been mixed [4]. A survey described responses given by patients when questioned about the cause of their non-adherence, which included forgetfulness (30\%), other priorities (16\%), dose omission (11\%), a lack of information (9\%), and emotional factors (7\%), but importantly $27 \%$ of the patients questioned did not provide any reason for 


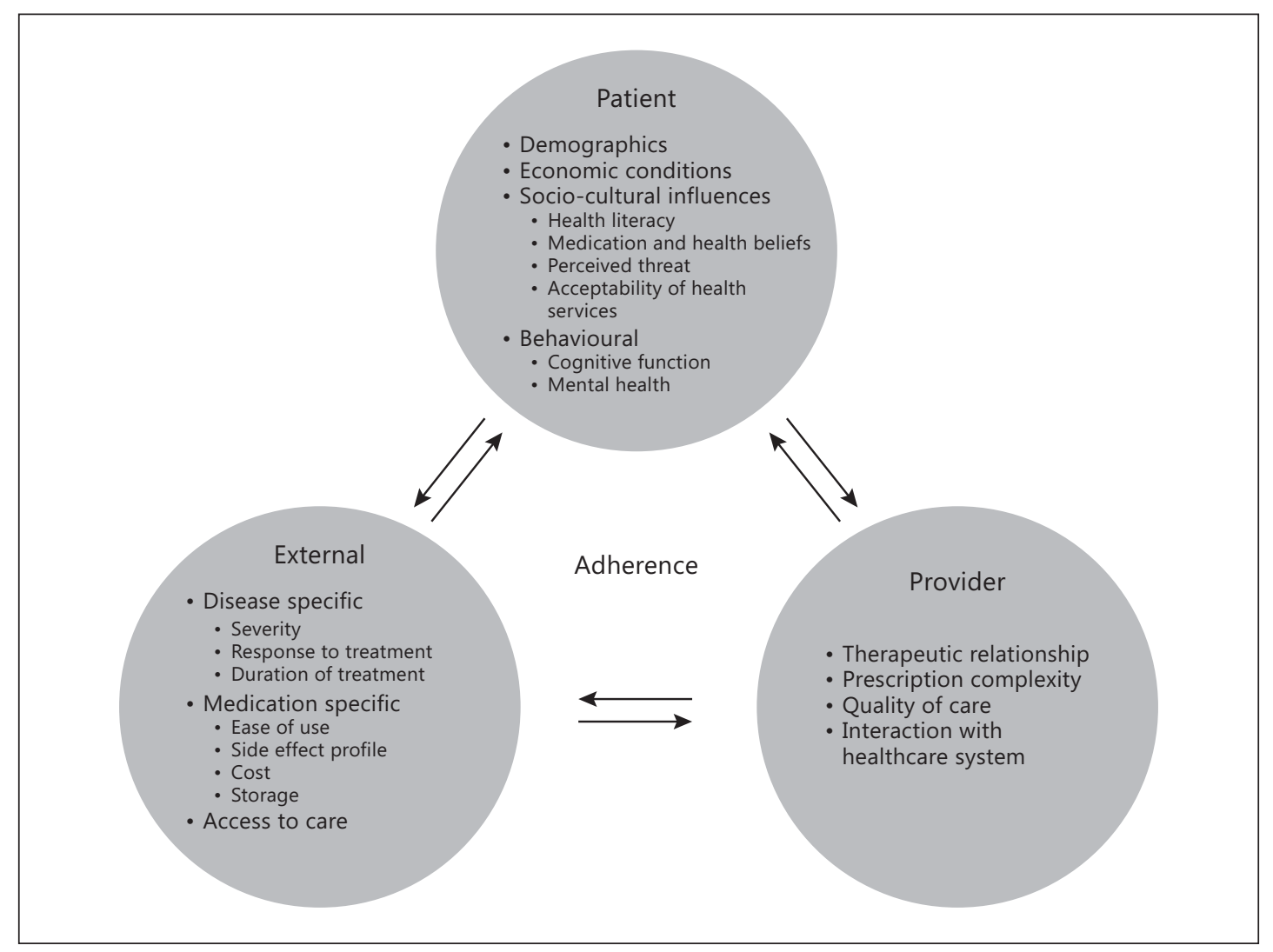

Fig. 1. Key contributing factors to treatment adherence $[3,5]$.

non-adherence [6]. The role HCPs can play in contributing to the likelihood of non-adherence is increasingly clear. Key examples are prescribing overly complicated treatment regimens, failing to explain the benefits versus side effects of a medication, not giving enough consideration to an individual's lifestyle, or having a poor therapeutic relationship with their patients $[5,7-9]$.

\section{Psychological Models Used to Improve Treatment Adherence}

Motivational interviewing is a psychological intervention that has generated a wide evidence base in recent years as an effective technique for improving psychological and physiological well-being [10]. Typically, a trained HCP will use a directive, patient-centred counselling style aimed at producing a change in behaviour by helping to facilitate the patient's exploration and resolution of their ambivalence. Motivational interviewing effectively helped facilitate a change in patient behaviour in a way that outperforms traditional advice-giving in approximately $80 \%$ of studies included in a recent meta-analysis [10]. Importantly, the nature of changes was almost always related to both adherence to prescribed medication and to the type of changes the patient makes in their lifestyle, such as diet. However, the availability of professionals trained in motivational interviewing is a barrier to its universal utilisation, and training in the technique is not routinely offered across professional disciplines.

Motivational interviewing is not the only intervention to increase treatment adherence. HCPs will recognise some of the following approaches: patient education, simplifying treatment routines, and considering the social issues that patients are facing and are willing to discuss. However, despite utilising these and other approaches, adherence rates remain 
variable. Some patients will stick rigidly to their treatment and some will be poor adherers, so what else do we need to consider?

Components of a patient's personality and temperament have been identified by some small studies as associating with their adherence rates in chronic diseases such as diabetes and asthma [11-13]. If we consider the specific contributory domain of personality to adherence, studies on patient personality tendency and the effectiveness of complimentary interventions would be useful in specific contexts. However, the evidence to date regarding the transferability of interventions suggests such specific studies would have limited value if we try to extrapolate them to a wider medical audience [2].

While we recognise that a person's tendency to react in a specific way in a given situation may be one small factor to consider in overall adherence, this, in combination with the recognised importance of the effective HCP-patient relationship, means there is the opportunity to make some gains in this area. Several health behaviour models have been developed to try and better understand and improve adherence [14, 15]. A simple, psychological model to enhance adherence that adds to the existing armamentarium, which can be widely understood and applied by non-psychologists and the range of HCPs that encounter patients during their treatment has the potential to be useful. Rubin's Four Tendencies model may provide an answer.

\section{Compliance, Concordance, Adherence, and "Rubin's Four Tendencies" Model}

We have consistently talked here of "adherence," a term which is now widely preferred [5]. "Compliance" is now less favoured due to its judgemental connotations [15] and the suggestion that the patient is simply passively following the doctor's orders. The concept of a compliant patient implies the treatment plan is not based on a "therapeutic alliance or contract established between the patient and the physician" [5]. "Concordance," a term that is also gaining acceptance in this area, refers to a patient-professional consultation that focuses on the process rather than specific patient behaviour, with an underlying ethos of shared decision-making as opposed to paternalism [16].

This move towards talking about "adherence" and "concordance" is evidence that we now implicitly recognise that this is a two-way process, and that patients have agency in their self-administered treatment. We would suggest that HCPs have expectations of their patients to engage in treatment, equally, patients have expectations of their treatment (whether positive or negative) and specific inner expectations for themselves regarding their engagement. Characterising these "external" and "internal" expectations, and the typical response patterns to them, is the aim of Rubin's Four Tendencies model, Gretchen Rubin's framework for understanding a person's responses to expectations [17].

In her recent publication (The Four Tendencies) and elsewhere, author Gretchen Rubin has described a model that identifies people as belonging to one of four dominant "Tendencies." Depending on how a person responds to outer and inner expectations, he or she falls into the category of "Upholder," "Questioner," "Obliger," or "Rebel” [17]. Hypothetically, when healthcare providers recognise a patient's Rubin Tendency, they would be better able to tailor medical expectations to that patient.

Rubin's model derives from the fact that we all face two kinds of expectations: outer expectations that others place on us (e.g. to meet a work deadline, to keep an appointment to have tests performed) and inner expectations that we place on ourselves (e.g. to keep a New Year's resolution, to eat more healthily, to "check my blood sugar more regularly"). Rubin's Four Tendencies model groups individuals into four distinct but overlapping domains based upon the way we react to outer and inner expectations (Fig. 2). 


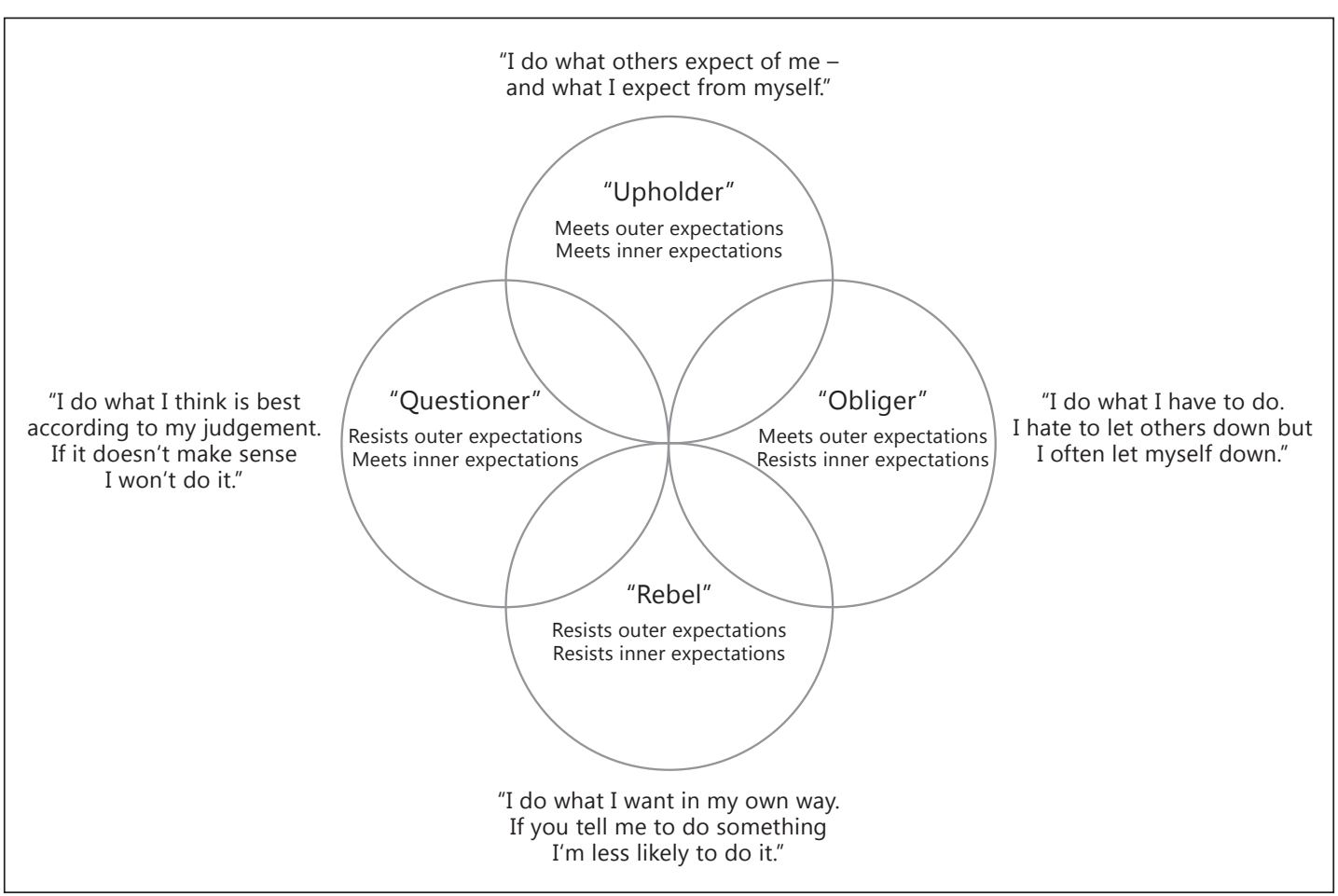

Fig. 2. Rubin's Four Tendencies.

We propose that a patient's Rubin Tendency may be a factor contributing to treatment adherence. In Figure 1, the Rubin Tendency would fall under the category "patient."

An "Upholder" responds readily to both outer and inner expectations, an "Obliger" responds readily to outer expectations but struggles to meet inner expectations. In contrast, a "Questioner" questions all expectations; Questioners meet an expectation only if they believe it's justified and, in effect, respond only to internal expectations (that is, expectations that meet their personal standard for justification). The final group, the "Rebel," resist all expectations, outer and inner alike.

How is a person's Rubin Tendency determined? In addition to creating a "flash evaluation" to quickly determine another person's Tendency through conversation [17], Rubin has also developed a freely available, online 13-item questionnaire to identify a person's dominant "Rubin Tendency." This is yet to be validated in the medical context, but within a nationally representative sample in the United States (July-August 2016, $n=1,564$ ), with variation in many demographic characteristics including geographical locations (ethnicity not captured), household income (education level not captured), age, and a mix of genders, the breakdown of tendencies was "Obliger" (41\%), "Questioner" (24\%), "Upholder" (19\%), and "Rebel" (17\%).

\section{How Might a Person's Rubin Tendency Present in the Clinical Context?}

Theoretically, people with a dominant "Upholder Tendency" would generally meet the expectations of the HCP and themselves to adhere to treatment.

Because people with a dominant "Questioner Tendency" likely require reasons and explanations in order to accept an expectation, and resist anything that appears arbitrary, inefficient, or unjustified, they would probably require robust explanations, answers, justifications, research, and data to validate any direction they are given, while also needing to respect 
the judgment and knowledge of the person giving the direction. "Questioners" also tend to want to customise whatever they're asked to do, to suit it to their individual needs.

Since people with a dominant "Obliger Tendency" readily meet outer expectations but struggle to meet inner expectations, they are likely to adhere far better when provided with outside accountability to meet health expectations such as monitoring, check-ups, and phone calls.

Since those with a dominant "Rebel Tendency" do what they choose to do, in their own way, they may typically respond better to a situation where their freedom is emphasised. They may respond well to a sequence where they have firstly been provided with information about a situation, then informed of the consequences of their actions or inactions, and then finally reminded that they can choose how to respond. In this group, it is important that they feel ownership of their choices without nagging or reminders, which can trigger their spirit of resistance (e.g. "you're not the boss of me, you can't tell me what to do"). People with a dominant Rebel Tendency often express their identity through their actions, so they may respond to reminders of "this is the person you choose to be" (e.g. "you choose to be athletic and energetic," "you choose to be a leader for others about ways to manage this condition," "cigarettes can't control someone as tough and independent as you").

Thus, when trying to understand reasons and possible solutions for non-adherence, "Rubin's Four Tendencies" model can provide a guide. The model would suggest that nonadherent people with a dominant "Questioner" Tendency were not convinced of a proposed treatment efficacy; this group would potentially respond to being given additional information, justifications, examples, and explanations that establish why adherence is crucial for their health. Dominant "Obligers" may have believed that no one will have noticed that they did not engage; for this group, because they do not easily meet inner expectations, it is essential that outer accountability (measures such as monitoring, check-ins, or reminders of the importance that they stay healthy for the good of their family) be put into place to strengthen any adherence issue. Individuals with a dominant "Rebel" Tendency likely did not appreciate being told what to do; for this group, "following doctor's orders" is a challenge, so they respond better when they are informed of what treatment they might choose to follow, in order to have the life they want (they want to be healthy, energetic, independent, pain-free, showing respect for their body, free themselves from the chains of addiction, etc.). The model suggests a person with a dominant "Upholder" Tendency is likely to engage with treatment; however, this does not establish they may not become non-adherent if a situational threshold is met (for example, situations of high psychological stress or previous bad experience of treatment).

This model suggests that it would be crucial to identify a patient's dominant "Rubin Tendency," because the strategies that work for a person with one Tendency may actually prove counter-productive for another. For instance, with a person who has a dominant "Obliger" Tendency, it may be helpful to emphasise accountability, monitoring, and supervision, but these measures may make it less likely that a person with a dominant "Rebel" Tendency will adhere.

It is important to note that Rubin's Four Tendencies model is not a mechanism for identifying potentially non-adherent patients. "Obligers," "Questioners," "Rebels," and even "Upholders" may all be adherent or non-adherent for different reasons.

The attractiveness of this simple model is that when it is applied to medical adherence, it could be easily used across therapeutic areas to tailor intervention strategies in a patientcentric way.

\section{Rubin's Four Tendencies in a Clinical Context}

Since first proposing the framework, Rubin has had contact informally from many HCPs about their experience applying the Four Tendencies model. For instance, a family physician 
Box 1. Case study: Helping a mother facilitate a strict diet for her child with phenylketonuria*

Gemma ${ }^{\dagger}$ is a mother of a 1-year-old boy with phenylketonuria who requires a strict low-phenylalanine diet, low-protein special foods, and amino acid supplements 3 times a day. Mum owns her own business and is used to doing things "her way." She is very pragmatic, has succeeded against the odds, and no health professional tells her "how to bring up her children." She struggled immediately with the diagnosis, and the first few months of management were challenging.

The mother particularly disliked the prescriptive nature of the dietary advice, lack of food choice, impact of the diet on family lifestyle, need for meal planning, and the rigid "step by step guidance" on how to feed her child.

Various strategies were used to help direct the child's treatment: she has been given different scenarios of outcome of patients with good and poor treatment, given information about the minimum she needed to do each day to manage blood phenylalanine levels, but also freedom in what she can do without direction. Importantly, she chooses how she fits treatment into her lifestyle (without value judgements from health professionals). We ensured that dietary information was readily available via Apps, websites, phone, email, and twitter. Regular contact was maintained with the mother to feedback blood results. The boy has excellent control of his condition and the mum now acts as role model and encourages other young mothers. She has done this without being asked.

* This case study is an anonymised summary of the treatment journey of a real mother and her child's treatment journey, published with their consent. ${ }^{\dagger}$ Not the mother's real name.

was treating a patient who needed to change her behaviour to lose weight and address prediabetes and insulin resistance - who was resistant to enact the doctor's recommendations. As the doctor spoke to the patient, he realised that she was displaying attitudes and behaviours associated with the "Rebel" domain, and he immediately changed his way of communicating. Rather than giving clear directions (his usual style, which he finds works best for him with most people), he gave her a list of suggestions to choose from, to try "if she wanted to." The patient did return for a later visit, and she'd followed one of his suggestions; she'd lost weight and was feeling much better. The doctor explained, "I don't think the result would have been the same had I been directive. That approach works with people I would consider to be Upholders and Obligers, and also Questioners, because I always give a detailed explanation of my recommendations. But for a person with the "Rebel" Tendency, that strategy is unlikely to work."

The case study described (Box 1) typifies an example of how Anita MacDonald (Author) has retrospectively applied Rubin's Four Tendencies model to further understand the successful approach that was taken with a parent who initially struggled with the management of her son's chronic condition. In hindsight, one might consider the mother to have been displaying behaviours associated with the "Rebel" Tendency, thus, Rebel-focused measures such as providing numerous choices and more autonomy to manage the dietary treatment providing biochemical control worked well.

\section{Position Statement}

Individuals respond to expectations in different but recognisable patterns, and we need to consciously take these into account when engaging patients in treatment programmes. Understanding the way in which a patient responds to external and internal expectations of 
treatment adherence using Rubin's Four Tendencies model might offer the opportunity to develop and validate simple clinical tools to match a patient's Rubin Tendency to a complementary specific intervention.

\section{Discussion}

To date, methods of improving medication adherence for chronic health problems are complex to administer and not universally effective [2]. There is need for a review of current evidence and a redoubling of efforts to develop effective and practical interventions to increase medical adherence; this article represents yet another "call to action" $[2,18]$ for HCPs to further escalate efforts to maximise potential treatment benefits during a time of increasing demands on healthcare resources.

We have proposed that identifying whether a patient has Tendencies that correlate with the domains of "Upholder," "Questioner," "Obliger," or "Rebel" according to Rubin's Four Tendencies model may influence the likelihood of specific interventions aimed at maximising adherence being effective.

We openly acknowledge that this is potentially just one of the approximately 100 recognised factors in the complex jigsaw that determines the likelihood of adherence [18]. Within the current context, where few effective and widely applicable tools are available, it is possible that in daily practice within patient groups with the same medical condition that are treated at a single centre, personality tendencies may become more apparent when explaining differences in adherence levels. We suggest this because the number of variables is likely reduced for such patient groups (e.g. patient education, convenience of administration, consequences of non-adherence are similar for all patients).

For HCPs in everyday practice, interacting with a non-adherent patient with a relatively stable socio-economic environment who has had other significant barriers mitigated, understanding the way they tend to react to internal and external expectations may be key in helping to change their adherence pattern.

\section{Testing the Four Tendencies Model in the Medical Setting}

Importantly, this model has the potential to exist inside the framework of an already established patient-professional relationship. When talking to a patient, we should always be trying to tailor our communications so they are understood and effective. The key question we are proposing HCPs ask themselves is: “Can we use Rubin's Four Tendencies model to tailor our communications so that we can work better with the patient to be adherent with their medication?"

Several questions require answering if interventions designed around Rubin's Four Tendencies model are to be validated and applied widely. We need to understand, firstly, if a person really has a dominant core Rubin Tendency, if their Tendency is stable over time and situation, and if a person's Rubin Tendency materially impacts upon the way that he or she engages with HCPs in a consistent manner.

\section{Future Research}

There are obviously potential strengths and weaknesses to any new model that is proposed, and several research questions will need to be answered before Rubin's Four Tendencies can be viewed as an effective tool for improving treatment adherence.

Firstly, the 13-item questionnaire will require validation in the clinical setting and across therapy areas. We would also then need for longitudinal studies to establish the stability of a person's dominant Rubin Tendency. The impact on adherence rates of recommending 


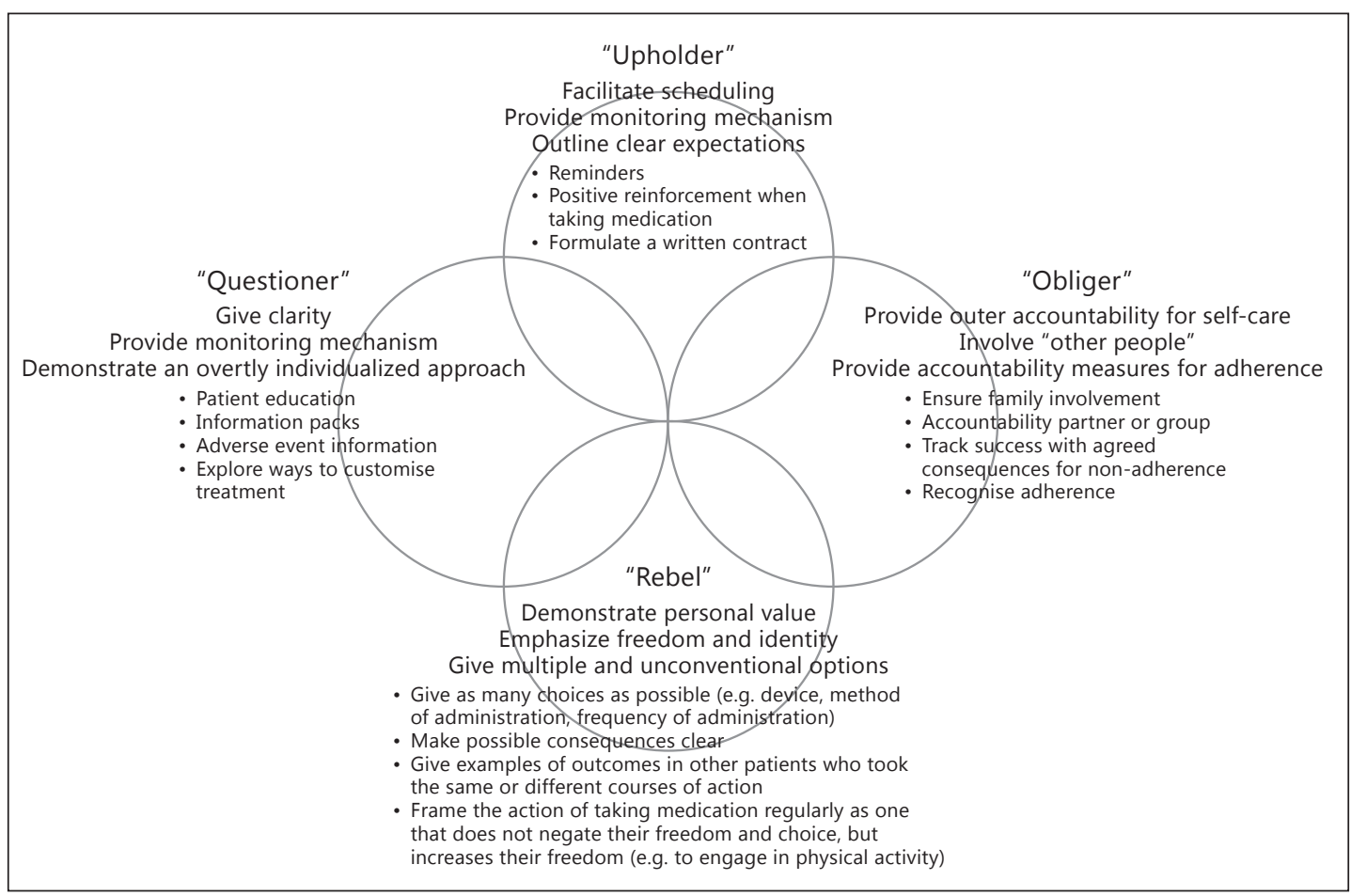

Fig. 3. Hypothetical alignment of adherence interventions to Rubin's Four Tendencies. Testing of the effectiveness of this mapping strategy should be performed using well-designed randomised controlled trials.

specific established interventions that are allocated according to a dominant baseline Rubin Tendency should then be prospectively investigated using clinical trials. Below are some examples of what such trials could investigate.

Proposal 1. Having a range of interventions available to increase adherence rates is already a recognised strategy [18]. A study that investigates the matching of interventions to Rubin Tendencies would be critical. For example, when setting up a treatment regimen, ensure there are a range of potential interventions in place to facilitate adherence, with options available that would appeal to individuals aligned to each of Rubin's Four Tendency groups. After establishing a person's baseline dominant Rubin Tendency, the patient could choose which adherence strategies they would like to undertake. Theoretically, when corrected against key variables such as socio-cultural considerations, and population demographics, this will allow the validation of interventions that are matched to each Rubin Tendency. A prespecified hypothesis of which dominant Rubin Tendency groups already established interventions may align against can be found in Figure 3.

Proposal 2. When a patient becomes non-adherent (or is classified as being at risk of nonadherence) when standard "first-line" interventions have already been implemented, consider choosing an already recognised "second-line" intervention based on their Rubin Tendency. A randomised controlled trial design would match "second-line" interventions to a patient's dominant Rubin Tendencies in the active group, while mismatched (but still established) interventions would be provided in the placebo group.

We can imagine several clinical scenarios in which the model would require testing. Would a person's dominant Rubin Tendency remain unchanged in in the face of acute treatment compared with long-term therapy for a chronic condition, such as insulindependent diabetes? Moreover, would the severity of the consequences of non-adherence 
(e.g. omitting insulin treatment) impact on the natural Tendency of a person's reactions to expectations?

We must acknowledge the limitations of any model centred on the HCP's approach, in that patient engagement, even at the start of treatment, is not guaranteed. A further example we see in our clinical practice where this model may be challenged is the patient presenting with extreme apathy. This patient likely has a complex history and multiple factors that must be considered, but we are presented with an interesting conundrum. Has our patient's apparent apathy presented because the first interventions were not tailored to their Tendency when reacting to expectations, fuelling their disengagement? A population of chronically non-adherent patients would be an interesting study population in which to test this model, as we could argue that the greater the disengagement with treatment, the more a patient needs an intervention that feels "effortless," and which reflects their natural Tendency.

We recognise that no model is universally applicable, and Rubin's Four Tendencies model is unlikely to be valid for all patients. It may be that interventions not typically aligned with a person's dominant Rubin Tendency will potentially work for that individual, and the utilisation of this model must be done within an open and collaborative "patient-professional" consultation. We believe such a model cannot become reductive and introduce the risk of "labelling" a patient. However, its attractiveness and potential is that it could allow HCPs the opportunity to simply choose a tailored and specific course of action that will be most effective for a particular patient early in the consultation process.

\section{Conclusion}

Increasing adherence to treatment is critical for improving outcomes and minimising healthcare resource waste. In general, several of the interventions available today are complex, resource intensive, expensive, and lack a firm focus on the patient [18]. We need an effective method to target the specific interventions that provide the most benefit to individual patients, and it is crucial that this method be easy and inexpensive to administer, and widely applicable, as part of everyday practice. Rubin's Four Tendencies model provides an opportunity to test such a targeted, patient-specific strategy. Such a tool is only useful if the interventions that are most effective for patients with a specific Rubin Tendency exist and can be implemented easily. In an environment with already stretched resources where the factors influencing adherence are complex and varied, the ability to tailor interventions to the patient is an important component of a wider problem.

\section{About the Authors}

Prof. Jeremy Kirk. I am a paediatric endocrinologist specialising in hormonal and specifically growth disorders. Adherence with growth hormone therapy, which has to be given by daily injection, is characteristically poor, and my own unit has pioneered patient choice for growth hormone device, one intervention which appears to improve adherence. The issues in non-adherence are however complex, and a simple tool which would enable interventions to be better targeted would be very useful.

Prof. Anita MacDonald. I am a paediatric nutrition specialist in Birmingham, UK. My daily practice involves asking children, and by extension their parents/carers, to adhere to strict and challenging diets to try and avoid the inevitable poor outcomes associated with rare metabolic diseases such as phenylketonuria. Adherence in my patients is incredibly important. 
The professionals in our team need a simple tool that they can use to help them best motivate our patients and, importantly, their parents when adherence becomes a challenge.

Paul Lavender. I have been involved in medical communications for almost 10 years. During this time, I repeatedly came across the topic of "adherence" as a constant across almost all medical fields; however, I saw little communication between those fields on best practices for improving adherence rates. I hope this article will encourage interdisciplinary dialogue and exchange on this important topic.

Jessica Dean. I am a Consultant Clinical Psychologist currently leading the Renal Psychology Service at Salford Royal Hospital. I have worked in the area of Clinical Health Psychology since qualifying in 2003. My research interests include: improving medical adherence using psychological interventions, the use of shared decision-making in renal services and the use of motivational interviewing in promoting medical adherence. I have published a number of papers on these topics and am a firm supporter of the need to personalise medical treatment and individualise patient care.

Gretchen Rubin. I am a writer who studies human nature. I've written several bestselling books, including Better Than Before and The Happiness Project; my latest book is The Four Tendencies. In my long study of human nature, happiness, and habit-formation, I constantly ask, "How and when can people change?" and I've discovered patterns that explain when and why people act - or why they don't act. When we understand how the Rubin Four Tendencies shape people's behaviour and attitudes, we can far more effectively help them adhere to treatment, exercise, or change their diet. Lacking understanding may actually make it less likely that they'll adhere to important recommendations.

\section{Acknowledgements}

Medical writing assistance for this article was provided by Dr. John Timney and funded by S. Karger Publishers, Switzerland. Paul Lavender gratefully acknowledges the inspiration and encouragement of Dr. Ekaterina Koledova and all of the team in the ECOS Study sponsored by Merck, Darmstadt, Germany.

\section{Disclosure Statement}

Jeremy Kirk is not aware of any potential conflict of interest.

Anita MacDonald has received research funding and honoraria from Nutricia, Vitaflo International, and Merck Serono. She is a member of the European Nutritionist Expert Panel (Biomarin), member of Sapropterin Advisory Board (Biomarin), member of the Advisory Board entitled ELEMENT (Danone-Nutricia), and member of an Advisory Board for Arla, Applied Pharma Research, and Sobi.

Jessica Dean declares no conflict of interest.

Paul Lavender is not aware of any potential conflict of interest.

Gretchen Rubin declares no conflict of interest. 
Kirk et al.: Can Treatment Adherence Be Improved by Using Rubin's Four Tendencies

Framework to Understand a Patient's Response to Expectations?

\section{References}

1 Brown MT, Bussell JK: Medication adherence: WHO cares? Mayo Clinic Proc 2011;86:304-314.

2 Nieuwlaat R, Wilczynski N, Navarro T, Hobson N, Jeffery R, Keepanasseril A, Agoritsas T, Mistry N, Iorio A, Jack S, Sivaramalingam B, Iserman E, Mustafa RA, Jedraszewski D, Cotoi C, Haynes RB: Interventions for enhancing medication adherence. Cochrane Database Syst Rev 2014:CD000011.

-3 Iuga A0, McGuire MJ: Adherence and health care costs. Risk Manag Healthc Policy 2014;7:35-44.

4 Fisher BG, Acerini CL: Understanding the growth hormone therapy adherence paradigm: a systematic review. Horm Res Paediatr 2013;79:189-196.

5 Osterberg L, Blaschke T: Adherence to medication. N Engl J Med 2005;353:487-497.

6 Cramer JA: Identifying and improving compliance patterns; in Cramer JA, Spilker B (eds): Patient Compliance in Medical Practice and Clinical Trials. New York, Raven Press, 1991, pp 387-392.

7 Elliott WJ, Maddy R, Toto R, Bakris G: Hypertension in patients with diabetes. Overcoming barriers to effective control. Postgrad Med 2000;107:29-32, 35-26, 38.

-8 Golin CE, Liu H, Hays RD, Miller LG, Beck CK, Ickovics J, Kaplan AH, Wenger NS: A prospective study of predictors of adherence to combination antiretroviral medication. J Gen Intern Med 2002;17:756-765.

$>9$ Ickovics JR, Meade CS: Adherence to antiretroviral therapy among patients with HIV: a critical link between behavioral and biomedical sciences. J Acquir Immune Defic Syndr 2002;31(suppl 3):S98-S102.

10 Rubak S, Sandbaek A, Lauritzen T, Christensen B: Motivational interviewing: a systematic review and metaanalysis. Br J Gen Pract 2005;55:305-312.

11 Axelsson M, Ekerljung L, Lundback B, Lotvall J: Personality and unachieved treatment goals related to poor adherence to asthma medication in a newly developed adherence questionnaire - a population-based study. Multidiscip Respir Med 2016;11:42.

12 Belvederi Murri M, Mamberto S, Briatore L, Mazzucchelli C, Amore M, Cordera R: The interplay between diabetes, depression and affective temperaments: a structural equation model. J Affect Disord 2017;219: 64-71.

13 Li X, Gao M, Zhang S, Xu H, Zhou H, Wang X, Qu Z, Guo J, Zhang W, Tian D: Medication adherence mediates the association between type $\mathrm{D}$ personality and high $\mathrm{HbA1}$ level in Chinese patients with type 2 diabetes mellitus: a six-month follow-up study. J Diabetes Res 2017;2017:7589184.

14 Jackson C, Eliasson L, Barber N, Weinman J: Applying COM-B to medication adherence: a suggested framework for research and interventions. Eur Health Psychol 2014;16:7-17.

15 Munro S, Lewin S, Swart T, Volmink J: A review of health behaviour theories: how useful are these for developing interventions to promote long-term medication adherence for TB and HIV/AIDS? BMC Public Health 2007; 7:104.

16 Dickinson D, Wilkie P, Harris M: Taking medicines: concordance is not compliance. BMJ 1999;319:787.

17 Rubin G: The Four Tendencies. New York, Harmony Books, 2017.

18 Bosworth HB, Granger BB, Mendys P, Brindis R, Burkholder R, Czajkowski SM, Daniel JG, Ekman I, Ho M, Johnson M, Kimmel SE, Liu LZ, Musaus J, Shrank WH, Whalley Buono E, Weiss K, Granger CB: Medication adherence: a call for action. Am Heart J 2011;162:412-424. 